\section{Expression of NKG2A inhibitory receptor on cytotoxic lymphocytes as an indicator of severity in Corona Virus Disease 2019 (COVID-19) patients}

\author{
Marwa M. Yasin ${ }^{1}$, Iman H. Shehata ${ }^{1}$, Nesma G. \\ Elsheikh $^{2}$ and Marwa S. Elsayed ${ }^{1}$
}

\footnotetext{
${ }^{1}$ Medical Microbiology \& Immunology Department, Faculty of Medicine, Ain Shams University, Cairo, Egypt.

${ }^{2}$ Geriatric \& Gerontology Department, Faculty of Medicine, Ain Shams University, Cairo, Egypt.
}

The Egyptian Journal of Immunology Volume 28 (3), 2021: 157-167. www.Ejimmunology.org
Corresponding author: Marwa S. Elsayed

Medical Microbiology \& Immunology Department, Faculty of Medicine, Ain Shams University, Abbasia square, Cairo, Egypt. E-mail:marshabban@yahoo.com.

\begin{abstract}
NK group 2 member A (NKG2A) receptor transduces inhibitory signaling; suppressing NK and T-cell cytokine secretion and cytotoxic function. This study aimed to assess the expression of NKG2A inhibitory receptor on natural killer (NK) cells and CD8+ T lymphocytes in COVID-19 patients and correlate the results with disease severity defined according to the criteria established by the world health organization, in a trial to understand the immunological response towards COVID-19 infection. The study enrolled 30 COVID-19 patients classified into 2 groups that comprised 15 subjects each; moderate and severe based on clinical, radiological, and laboratory findings. Ten age and sex matched apparently healthy individuals were included in this study as a control group. About $1 \mathrm{ml}$ EDTA anti-coagulated blood samples were collected for measuring expression of NKG2A/CD159a on CD56+ CD3- NK and CD3+CD8+ T cells by flow cytometry. Results revealed that COVID-19 patients had significantly lower NK and CD8+ T cell counts compared to healthy subjects. Severe cases had significantly lower CD8+ T counts compared to moderate ones. Percentages of NK and CD8+T cells expressing NKG2A receptor were significantly higher in cases compared to controls. Comparison between severe and moderate cases revealed that although the percentages of NK cells expressing NKG2A receptor were not significantly higher in severe cases, the mean fluorescence intensity was significantly higher. The percentages of $C D 8+T$ cells expressing NKG2A receptor were significantly higher in severe cases with higher mean fluorescence intensity. In conclusion, our results indicate that elevated NKG2A expression on cytotoxic lymphocytes correlates with disease severity in COVID19 patients, and may serve as a potential marker for prognosis. Additionally, the blockade of NKG2A should be investigated as means of enhancing NK cell and cytotoxic T cells antiviral immunity in patients with severe COVID-19 infection.
\end{abstract}

Keywords: NKG2A, NK cells, CD8+T cells, Flow cytometry, COVID-19. 


\section{Introduction}

Coronavirus disease 2019 (COVID-19) is an arising viral infection caused by severe acute respiratory syndrome coronavirus 2 (SARS-CoV2), a novel beta coronavirus firstly recognized in Wuhan City, Hubei Province, China during a burst of respiratory illness cases. ${ }^{1}$ After few weeks of onset, the disease turned into a worldwide pandemic with 111,102,016 cases and 2,462,911confirmed deaths as reported by the World Health Organization on 22 February $2021 .^{2}$

This novel virus is associated with higher mortality than other respiratory viruses; it additionally shows a more extensive variety in its clinical presentation. ${ }^{3}$ Most of COVID-19 cases (about $80 \%$ ) is asymptomatic or display mild to moderate symptoms, however roughly the $15 \%$ advance to serious pneumonia and about $5 \%$ develop the critical disease with respiratory failure and organ dysfunction. ${ }^{4}$

Innate immunity is the first line of defense against virus invasion. Upon recognition of pathogen associated molecular patterns, cytolytic immune responses are initiated, mainly through type I interferons and NK cells. Adaptive immunity likewise has a significant role in viral elimination via activated cytotoxic $T$ cells that kill virus-infected cells and antibodyproducing $B$ cells that target virus specific antigens. $^{5}$

NKG2A/CD159a is an immunoreceptor tyrosine-based inhibitory motif (ITIM)-bearing receptor expressed on both $\mathrm{T}$ and NK cells. Roughly $50 \%$ of peripheral blood NK cells express NKG2A and its expression can be upregulated upon stimulation with cytokines, such as interleukin-15. In healthy individuals, around $5 \%$ of human peripheral blood CD8+T cells express cell-surface NKG2A at steady state; however this expression can be upregulated by chronic antigenic stimulation. ${ }^{6}$ NKG2A belongs to a family of lectins, which forms a heterodimer with CD94, another NK cell expressed C-type lectin, otherwise called Killer Cell Lectin Like Receptor D1 (KLRD1). The NKG2A/CD94 complex binds to the non-classical MHC I molecule, HLA-E in humans and transduces inhibitory signals, which suppress NK and CD8+ ells activity.

A recent study showed that NKG2A expression is increased on NK cells and CD8+ T lymphocytes whose functions were suppressed, a picture consistent with $\mathrm{T}$ cell exhaustion. Moreover, the counts of NK and CD8+ T cells increased in recovering patients, while expression of NKG2A decreased. The authors suggested that NKG2A over-expression can be a mechanism via which SARS-CoV-2 supersedes the immune system of the host, resulting in functional exhaustion of the immune response against the virus. ${ }^{8}$

This study aimed to assess the expression of NKG2A inhibitory receptor on NK cells and CD8+ $T$ lymphocytes in COVID-19 patients and correlate the results with severity of the disease, in a trial to understand the immune response and open a new aspect in the management of COVID-19 infection using monoclonal antibodies targeting this receptor.

\section{Subjects and Methods}

\section{Subjects}

This observational cross-sectional study was conducted on 30 COVID-19 patients admitted to the El-Demerdash Geriatrics isolation Hospital, during the period from September to November 2020. They were diagnosed on clinical, radiological, and laboratory basis. Patients were classified into 2 groups; group A: 15 moderate cases, and group B: 15 severe cases. Ten age and sex matched apparently healthy individuals were also included in this study as a control group (group C).

Patients were classified into moderate and severe disease according to the following criteria; by definition moderate cases: had fever and other respiratory symptoms with pneumonia manifestation through image results, and severe cases: had any one of the following: respiratory distress, hypoxia ( $\mathrm{SpO} 2 \leq$ 93\%), abnormal blood gas analysis: ( $\mathrm{PaO} 2<$ $60 \mathrm{mmHg}, \mathrm{PaCO} 2>50 \mathrm{mmHg}) .{ }^{9}$ Patients were excluded if they had chronic infections (e.g. $\mathrm{HCV}, \mathrm{HBV}$ ), cancers or any other immunological disorders. 
The study was approved by the Ethical Committee of Ain Shams University Hospitals, and an informed consent was taken directly from the patients or their first degree relatives for sample collection. Relevant clinical and demographic data were collected using a standardized data collection form.

\section{Flow cytometry}

About $1 \mathrm{ml}$ EDTA anti-coagulated blood samples were collected for measuring expression of NKG2A/CD159a on NK and CD8+ T cells by flow cytometry. A lyse solution (Al-Gomhoreya CA, Egypt) and the following monoclonal antibodies (mAbs) were used: R- Phycoerythrin-Cyanine 7 (PC7) -conjugated anti- CD159a, PhycoerythrinCyanine 5 (PC5) -conjugated anti- CD3, Rphycoerythrin (R-PE)-conjugated anti-CD56, and fluorescent isothiocyanine (FITC) -conjugated anti- CD8. All mAbs were purchased from Beckman Coulter (Hialeah, FL). Navios six colors flow cytometer (Beckman Coulter, USA) employing a 4-color panel was used to determine fluorescence intensity. For every subject, a single tube contained the four mentioned mAbs ( $5 \mu \mathrm{L}$ of each) added to $50 \mu \mathrm{L}$ of EDTA treated blood. The tubes were mixed well using vortex mixer and incubated for 15 $\mathrm{min}$ at room temperature in the dark. Two $\mathrm{ml}$ of lyse solution were added and mixed using vortex mixer thoroughly. The tubes were further incubated for 5-10 minutes at room temperature in the dark.

Sample acquisition was set to acquire 10,000 events. Lymphocytes were gated according to light scatter parameters that reflect cell morphological characteristics (forward scatter reflecting cell size, side scatter reflecting internal structure of cell). The NK cells were identified as the CD3-CD56+ cell population and referred to as W. Similarly cytotoxic T cells were identified as CD3+CD8+ lymphocytes and referred to as $V$ (fig.1). The expression of NKG2A /CD159a on each of the gated NK and CD8+ T cell populations was measured using PC7 conjugated anti- CD159a, respectively (fig.2). Data was analyzed with Navios software version 1.1.

\section{Statistical analysis}

All results are presented as mean and standard deviation (SD) values. Continuous variables were compared between 2 groups using Student $\mathrm{t}$ test or the Mann-Whitney $U$-test, depending on the distribution of raw data. Spearman's correlation was used to assess the correlation between continuous variables. A significance level of $P<0.05$ was used in all tests. All statistical procedures were carried out using SPSS version 15 for Windows (SPSS Inc, Chicago, IL, USA).

\section{Results}

Thirty COVID -19 patients were enrolled in this study. They included 17 males and 13 females. The age of patients ranged from 24 to 70 years. They were classified to 2 groups; moderate cases: 7 males and 8 females with mean age $48.07 \pm 12.52$ years, and severe cases: 10 males and 5 females with mean age $47.20 \pm 10.52$ years. Ten age and sex matched apparently healthy individuals which included 5 males and 5 females with mean age $44.10 \pm 12.32$ years, were included in this study as a control group.

Comparison of laboratory results revealed statistically significant differences between COVID -19 patients and the apparently healthy control group as regards total leukocyte count, neutrophil count, absolute lymphocyte count, and neutrophil/ lymphocyte ratio as shown in table (1). 
Table 1. Comparison of laboratory findings between COVID -19 patients and controls.

\begin{tabular}{|c|c|c|c|}
\hline & $\begin{array}{l}\text { COVID -19 patients } \\
\quad(\text { Mean } \pm \text { SD) }\end{array}$ & $\begin{array}{l}\text { Apparently healthy controls } \\
\text { (Mean } \pm \text { SD) }\end{array}$ & $P$ value \\
\hline Hemoglobin (g/dl) & $12.02 \pm 1.92$ & $12.67 \pm 0.84$ & NS* \\
\hline Platelet count $\left(* 10^{\wedge} 3\right)$ & $224.73 \pm 96.15$ & $258 \pm 25.14$ & NS** \\
\hline Total Leukocyte Count $\left(* 10^{\wedge} 3\right)$ & $10.55 \pm 5.03$ & $5.76 \pm 1.73$ & $0.006 *$ \\
\hline Neutrophil count $\left({ }^{*} 10^{\wedge} 3\right)$ & $8.65 \pm 5.02$ & $4.32 \pm 1.03$ & $0.011^{*}$ \\
\hline Absolute lymphocyte count $\left({ }^{*} 10^{\wedge} 3\right)$ & $0.98 \pm 0.4$ & $2.33 \pm 0.46$ & $0.001 *$ \\
\hline Neutrophil/Lymphocyte Ratio & $10.99 \pm 9.64$ & $1.89 \pm 0.46$ & $0.001^{* *}$ \\
\hline
\end{tabular}

[Ungated] SS INT LOG/FS INT LIN

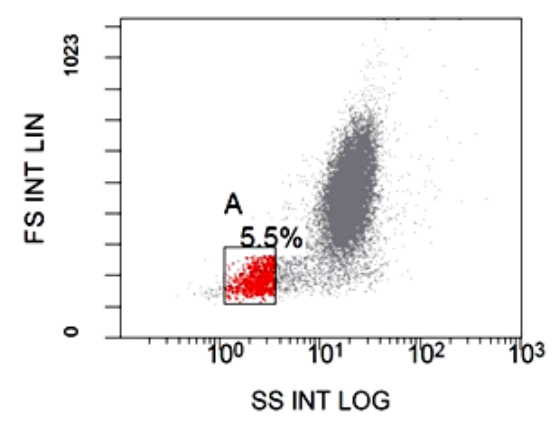

(A) FLA INT LOG/FL1 INT LOG

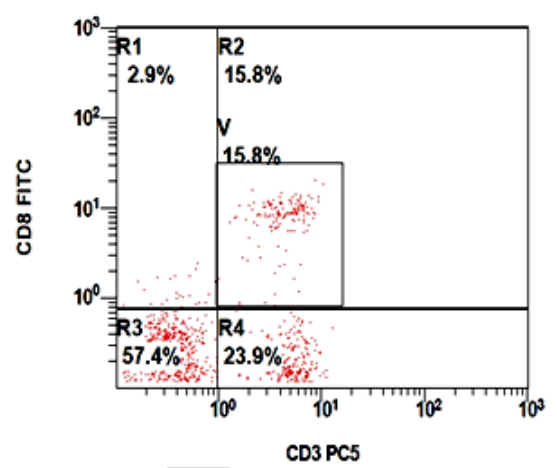

B
[A] FL4 INT LOG/SS INT LOG
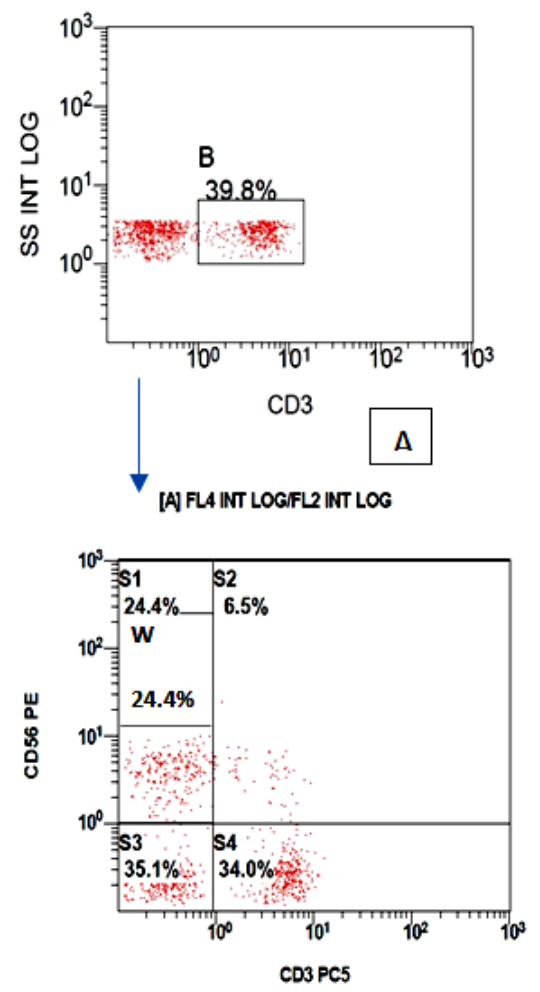

C

Figure 1. Flow cytometric gating and analysis of CD8+ T cells and NK cells from a representative COVID-19 patient. (A)Gating on lymphocytes, (B) The upper right quadrant represents CD8+Tcells (CD3+CD8+) referred to as V , (C) The upper left quadrant represents NK cells (CD3-CD56+) referred to as W. 
[A AND V] FL5 INT LOG

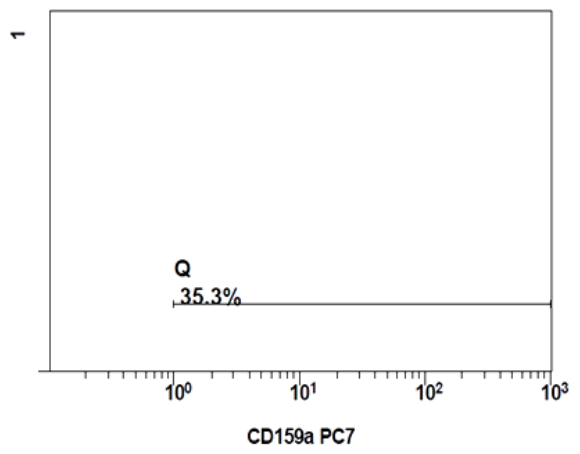

A
[A AND W] FL5 INT LOG

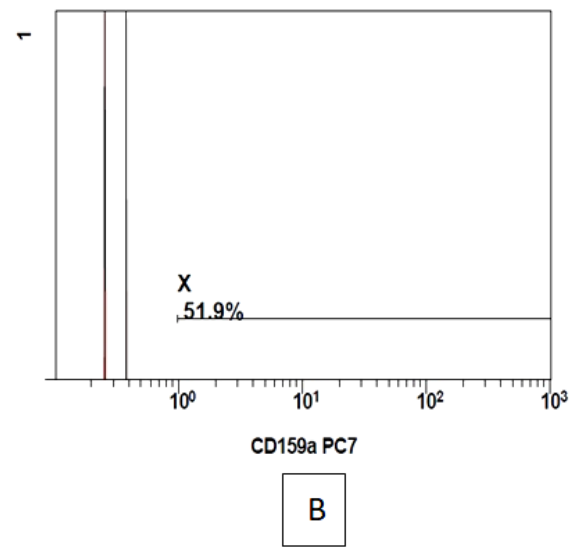

Figure 2. Flow cytometric analysis of NKG2A expression by CD8+ T and NK cells from a representative COVID-19 patient. (A) The expression of NKG2A/CD159a (was referred to as A) is measured on the CD3+CD8+cells, (B) the expression of NKG2A/CD159a is measured on the CD3-CD56+cells.

The study revealed lower NK cell percentages (mean \pm SD $8.46 \pm 5.84$ vs $13.5 \pm 3.57$ ) and counts (mean \pm SD $78.47 \pm 51.26$ vs $304.8 \pm$ 53.35) in COVID19 cases compared to healthy individuals, and the difference between them was statistically highly significant. COVID-19 patients had significantly higher percentage of NK cells expressing NKG2A receptor compared to controls (mean \pm SD $44.72 \pm 17.74$ vs $23.4 \pm$ $5.8)$, with higher mean fluorescence intensity (mean \pm SD $5.64 \pm 2.1$ vs $2.5 \pm 0.47$ ) (table 2).

COVID-19 patients had lower CD8 +T cell counts compared to the control group (mean \pm
SD $218.97 \pm 133.49$ vs $472.5 \pm 76.03$ ) and the difference between them was of high statistical significance. The percentage of $\mathrm{CD} 8+\mathrm{T}$ cells expressing NKG2A receptor was higher in cases compared to the control group with high statistical significance (mean \pm SD $11.9 \pm 10.4$ vs $2.1 \pm 1.1$ ). Although the mean fluorescence intensity was higher in the COVID-19 patients compared to the control group the difference was not statistically significant (mean \pm SD $3.8 \pm$ 2.1 vs $2.6 \pm 0.4$ ) (table 2 ).

Table 2. Comparison of NK and CD8+ T cell frequencies, counts, and markers measured between COVID -19 patients and controls.

\begin{tabular}{lccc}
\hline & $\begin{array}{c}\text { COVID }-19 \text { patients } \\
\text { (Mean } \pm \text { SD) }\end{array}$ & $\begin{array}{c}\text { Apparently healthy controls } \\
\text { (Mean } \pm \text { SD) }\end{array}$ & $P$ value \\
\hline NK cell \% & $8.46 \pm 5.84$ & $13.5 \pm 3.57$ & $0.001^{* *}$ \\
NK cell count (cells/ $\mu \mathrm{L}$ ) & $78.47 \pm 51.26$ & $304.8 \pm 53.35$ & 0.001 \\
NKG2A NK-cell\% & $44.72 \pm 17.74$ & $23.4 \pm 5.8$ & $0.001^{* *}$ \\
NKG2A NK-cell MFI & $5.64 \pm 2.1$ & $2.5 \pm 0.47$ & $0.001^{* *}$ \\
\hline CD8 +T cell \% & $21.18 \pm 7.4$ & $13.5 \pm 3.57$ & NS* $^{*}$ \\
CD8 +T cell count (cells/ $\mu \mathrm{L})$ & $218.97 \pm 133.49$ & $304.8 \pm 53.35$ & $0.001^{* *}$ \\
NKG2A CD8+T cell\% & $11.9 \pm 10.4$ & $23.4 \pm 5.8$ & $0.001^{* *}$ \\
NKG2A CD8 +T cell MFI & $3.8 \pm 2.1$ & $2.5 \pm 0.47$ & NS* $^{*}$ \\
\hline
\end{tabular}

*Student $\mathrm{t}$ test, **Mann Whitney test. $P$ values indicate differences between COVID -19 patients and apparently healthy controls; $\mathrm{MFI}=$ Mean Fluorescence Intensity, $P$-value of $>0.05$ is not significant (NS). 
The group of patients with severe disease had lower haemoglobin level, platelet counts, and absolute lymphocyte counts compared to moderate ones and the differences were statistically significant. The neutrophil count and the neutrophil/lymphocyte ratio were higher in severe cases, than moderate cases, but the differences were of no statistical significance. The group of severe cases had significantly higher lactate dehydrogenase (LDH), D-dimer and inflammatory markers including C-reactive protein (CRP) and ferritin compared to moderate cases (table 3).

Table 3. Comparison of laboratory findings between moderate and severe COVID-19 cases.

\begin{tabular}{|c|c|c|c|}
\hline & $\begin{array}{c}\text { Moderate cases } \\
\text { (Mean } \pm \text { SD) }\end{array}$ & $\begin{array}{l}\text { Severe cases } \\
\text { (Mean } \pm \text { SD) }\end{array}$ & $P$ value \\
\hline Hemoglobin (g/dl) & $12.82 \pm 1.13$ & $11.23 \pm 2.24$ & NS* \\
\hline Platelet count $\left(* 10^{\wedge} 3\right)$ & $261.33 \pm 108.29$ & $188.13 \pm 67.46$ & $0.035^{*}$ \\
\hline Total Leukocyte Count $\left(* 10^{\wedge} 3\right)$ & $11.15 \pm 4.43$ & $9.95 \pm 5.66$ & NS* \\
\hline Neutrophil count $\left(* 10^{\wedge} 3\right)$ & $8.64 \pm 4.51$ & $8.65 \pm 5.65$ & NS* \\
\hline Absolute Lymphocyte count $\left({ }^{*} 10^{\wedge} 3\right)$ & $1.25 \pm 0.35$ & $0.72 \pm 0.26$ & $0.001^{*}$ \\
\hline Neutrophil/Lymphocyte Ratio & $8.01 \pm 5.53$ & $13.98 \pm 11.95$ & NS** \\
\hline Serum ferritin (ng/ml) & $488.2 \pm 207$ & $1105.9 \pm 402.1$ & $0.001 * *$ \\
\hline D-dimer (mg/L) & $0.5 \pm 0.2$ & $3.4 \pm 1.9$ & $0.001^{*}$ \\
\hline Lactate dehydrogenase (IU/L) & $346.5 \pm 65.5$ & $547.8 \pm 75.7$ & $0.001^{*}$ \\
\hline C-reactive protein (mg/dl) & $4.3 \pm 1.5$ & $17.7 \pm 8.9$ & $0.001^{*}$ \\
\hline
\end{tabular}

*Student $t$ test, **Mann Whitney test. P values indicate differences between moderate and severe COVID -19 cases; $P>0.05$ is not significant (NS).

The results showed lower NK cell \% (mean \pm SD $6.95 \pm 6.16$ vs $9.97 \pm 5.28$ ) in the group of severe cases compared to moderate ones, but the difference was not statistically significant. NK cell counts were also lower in the group of severe cases (mean \pm SD $43.87 \pm 30.61$ vs $113.07 \pm 44.05$ ), and the difference was statistically highly significant. Although the percentage of NK cells expressing NKG2A receptor was higher in group of severe cases compared to the group of moderate cases (mean \pm SD $50.73 \pm 18.96$ vs $38.72 \pm 14.65$ ), the difference was not statistically significant. The mean fluorescence intensity was higher in the group of severe and critical cases (mean \pm SD $7.07 \pm 2.1$ vs $4.21 \pm 0.58$ ) with a high statistical significance (table 4).
The severe cases group showed lower CD8 $+T$ cell \% (mean \pm SD $19.85 \pm 5.14$ vs $22.51 \pm 9.11$ ) but the difference was not statistically significant. However, the CD8 $+\mathrm{T}$ cell counts were significantly lower in severe cases (mean \pm SD $149.53 \pm 83.39$ vs $288.4 \pm 140.09$ ). The severe cases had significantly higher percentages of CD8 $+T$ cells expressing NKG2A receptor (mean \pm SD $18.3 \pm 10.5$ vs $5.5 \pm 5$ ) with higher mean fluorescence intensity (mean \pm SD $5 \pm 2$ vs $2.7 \pm 1.6$ ) (table 4).

We examined the correlation between NKG2Aexpression on CD8+T cells and NK cells among all participants and found a significant correlation ( $r=0.412, P=0.008$; Fig. 3 ). 
Table 4. Comparison of NK and CD8+T cell frequencies, counts, and markers measured between moderate and severe COVID-19 cases.

\begin{tabular}{lccc} 
& $\begin{array}{c}\text { Moderate cases } \\
\text { (Mean } \pm \text { SD) }\end{array}$ & $\begin{array}{c}\text { Severe cases } \\
\text { (Mean } \pm \text { SD) }\end{array}$ & $P$ value \\
\hline NK cell \% & $9.97 \pm 5.28$ & $6.95 \pm 6.16$ & NS** \\
NK cell count (cells/ $\mu \mathrm{L}$ ) & $113.07 \pm 44.05$ & $43.87 \pm 30.61$ & $0.001^{* *}$ \\
NKG2A NK-cell\% & $38.72 \pm 14.65$ & $50.73 \pm 18.96$ & NS** \\
NKG2A NK-cell MFI & $4.21 \pm 0.58$ & $7.07 \pm 2.1$ & $0.001^{* *}$ \\
\hline CD8 +T cell \% & $22.51 \pm 9.11$ & $19.85 \pm 5.14$ & NS* \\
CD8 +T cell count (cells/ $\mu \mathrm{L}$ ) & $288.4 \pm 140.09$ & $149.53 \pm 83.39$ & $0.003^{*}$ \\
NKG2A CD8+ T cell\% & $5.5 \pm 5$ & $18.3 \pm 10.5$ & $0.001^{* *}$ \\
NKG2A CD8 +T cell MFI & $2.7 \pm 1.6$ & $5 \pm 2$ & $0.002^{* *}$ \\
\hline
\end{tabular}

*Student $\mathrm{t}$ test, ${ }^{* *}$ Mann Whitney test. $\mathrm{P}$ values indicate differences between moderate and severe COVID -19cases; $\mathrm{MFI}=$ Mean Fluorescence Intensity, $P>0.05$ is not significant (NS).

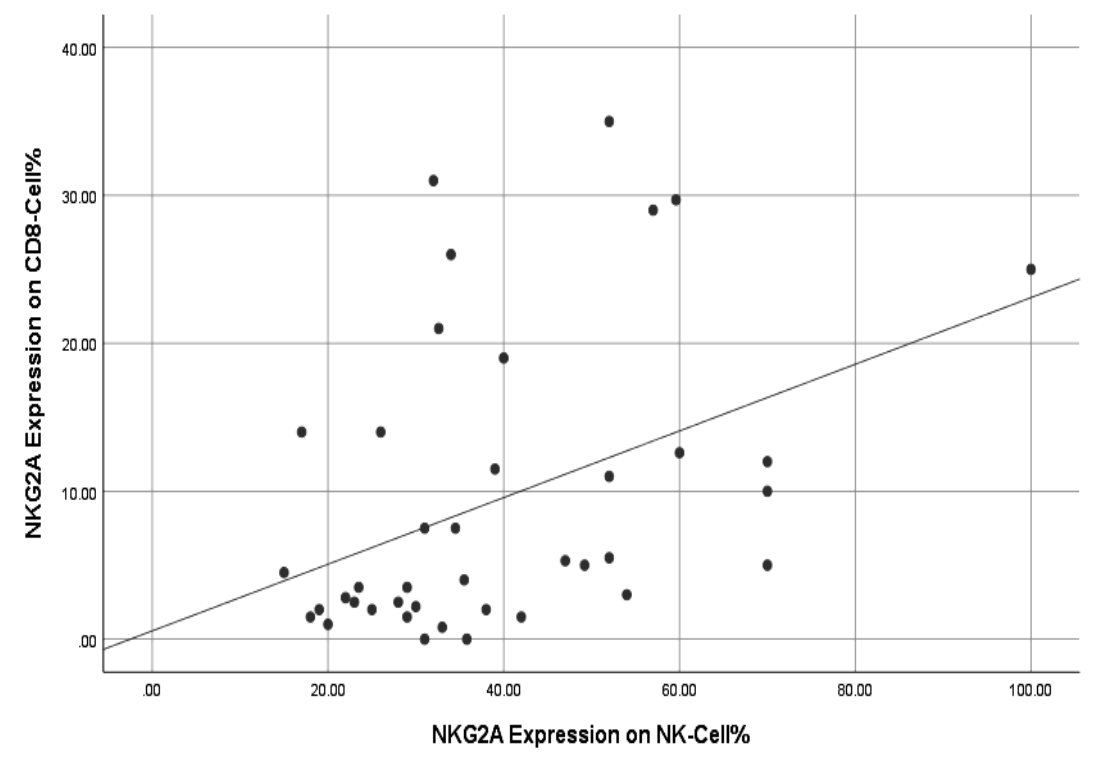

Figure 3. Scatter diagram showing a significant correlation between NKG2A Expression on CD8+ T cells and NKG2A Expression on NK-cells of COVID-19 patients. $(r=0.412, P=0.008)$.

\section{Discussion}

The host immune system is involved in the pathogenesis of COVID-19. Cytotoxic lymphocytes such as CD8+ T lymphocytes and NK cells play a major role in the control of viral infections and viral clearance. This could be mediated by direct cytotoxicity and release of IFN- $\gamma$. However, in some COVID-19 patients, it was reported that $\mathrm{CD} 8+$ and NK cells showed alterations in terms of count and impaired function. ${ }^{10}$ To our knowledge, this is the first reported investigation of a possible role of elevated NKG2A expression on cytotoxic lymphocytes as an indicator of disease severity among Egyptian patients with COVID-19.

Several studies highlighted relevant changes in innate and adaptive immune responses in COVID-19 patients. Specifically, lymphocytopenia and modulation of total neutrophils are common hallmarks and appear to be directly correlated with disease severity and death. ${ }^{11,12,13,14,15,16}$ Our results revealed that COVID-19 patients had higher total leukocyte 
counts, neutrophil counts, and neutrophil/ lymphocyte ratio but lower absolute lymphocyte counts compared to healthy subjects. Furthermore, the group of severe and critical cases had lower haemoglobin level, platelet counts, and absolute lymphocyte counts compared to moderate cases. Possibly such a reduction of lymphocytes might be secondary to a massive release of inflammatory mediators as a response to infection. Alternatively, failure to observe lymphocytes in the blood might be due to their homing or recruitment to the lung or other infected organs. ${ }^{17}$ The impaired lymphocytes in COVID19 patients may easily lead to microbial infection, further promoting activation and recruitment of neutrophils in the blood of patients. ${ }^{18}$ We observed that severe cases had higher LDH, D-dimer and inflammatory markers including CRP and ferritin compared to moderate cases, which agreed with previous reports . ${ }^{19,20,21,22}$

This study showed lower NK cell frequency and counts in the COVID-19 patients in comparison to healthy controls. Moreover, NK cell counts were lower in severe cases than moderate ones. This agreed with several emerging studies.$^{23,24,25,26}$ NK cells are nonresident lung cells; but they can reach lungs from peripheral blood via chemokine receptor CXCR3 binding to chemoattractant ligands secreted by monocytes and macrophages in lungs of COVID-19 patients. $^{27}$ Several upregulated genes in peripheral blood mononuclear cells of COVID-19 patients are involved in apoptosis and P53 signalling pathways, suggesting that lymphopenia could be due to SARS-CoV-2-mediated apoptosis. ${ }^{28}$

We observed that percentages of NK cells expressing NKG2A receptor were higher in COVID-19 cases compared to controls, with higher mean fluorescence intensity. We didn't find a significant difference between severe and moderate cases, but the mean fluorescence intensity was significantly higher. Our results agreed with Zheng et al., who found that percentages of peripheral NK cells expressing the inhibitory marker NKG2A were higher in COVID-19 patients than healthy controls and suggested functional exhaustion of peripheral
NK cells during SARS-CoV-2 infection and disease progression as an explanation to their findings. Furthermore, numbers of NK cells were restored with reduced expression of NKG2A in patients convalescing after therapy. ${ }^{8}$ Demaria et al. found that the NKG2A expressing NK cells were lower in patients with acute respiratory distress syndrome (ARDS), although the cell surface density of NKG2A was upregulated in this group denoting that its upregulation is associated with disease severity. In addition, high levels of NKG2A expression were also observed in NK cells isolated from the bronchoalveolar lavage fluid of ARDS COVID-19 patients, and these levels were even higher than those observed in blood cells of the patients tested. ${ }^{29}$ Carvelli et al. analysed myeloid and lymphoid populations by immunophenotyping of blood and bronchoalveolar lavage fluid (BALF) in 10 healthy controls, 10 pauci-symptomatic COVID19 patients, 34 pneumonia patients, and 28 patients with ARDS due to SARS-CoV-2 and found that the proportion of mature NK cells were reduced in patients with ARDS and NK cells showed increased NKG2A, PD-1, and CD39 expression. $^{30}$ This upregulated NKG2A expression on NK cells was found to be mediated by the viral spike protein. In vitro coculture of peripheral NK cells with SARS-CoV2 spike protein transfected lung epithelial cells resulted in diminished NK degranulation and NKG2A up-regulation. ${ }^{31}$ In light of our results and other reports anti- NKG2A therapy could be investigated; as a possible solution for COVID-19 patients to restore NK cells and their function. $^{32,33 .}$

Several authors reported that CD8+ T cells were drastically decreased in severe COVID-19 cases. $^{26,34,35,36}$ In this regard, we observed that COVID-19 patients had lower CD8 +T cell count compared to controls, and counts were lower in severe cases in comparison to moderate ones. It remains unclear if this is due to trafficking of CD8+ $\mathrm{T}$ cells into tissues with ongoing SARSCoV-2 replication, increased elimination of CD8+ $\mathrm{T}$ cells during COVID-19, or pre-existing abnormally low levels of $\mathrm{CD} 8+\mathrm{T}$ cells in individuals who experience severe disease. ${ }^{37} \mathrm{We}$ demonstrated that percentages of $\mathrm{CD} 8+\mathrm{T}$ cells 
expressing NKG2A receptor were higher in COVID-19 patients compared to healthy controls. Furthermore, elevated NKG2A expression on CD8 $+\mathrm{T}$ cells correlated with disease severity. Previous studies observed similar findings. ${ }^{8,30}$

A significant correlation between NKG2A expression on CD8+T cells and NK cells among all participants was observed in this study. NKG2A is a heterodimeric inhibitory receptor expressed prominently by cytotoxic lymphocytes, such as NK cells and CD8+ T cells. Upon binding of this receptor to the nonclassical HLA-E molecule, inhibitory signals are transduced, resulting in suppression of the cytotoxic activity of these immune cells, and promoting viral spreading during a variety of chronic viral infections. ${ }^{38}$ Expression of the inhibitory marker NKG2A leads to reduced expression of IFN $\gamma$, IL-2 and TNF $\alpha$ as well as granzyme B levels. ${ }^{39}$ Additionally, NKG2A expression on NK cells and $T$ cells could be a critical factor in the immune dysfunction of COVID-19 patients, disturbing the crosstalk between these immune cells and neutrophils. The IFN- $\gamma$ secreted by activated NK cells has a dual role as an antiviral cytokine, at the same time it is also important for impairment of neutrophil expansion and survival in the lungs, thus regulating and limiting neutrophil mediated tissue injury. ${ }^{40,41}$ The increased NKG2A expression on NK cells provides inhibitory signals, which in turn inhibits NK cell expansion, while the neutrophil numbers remain unrestrained. This is made more serious by the inflammatory environment established by the cytokine release syndrome, particularly IL-6 and IL-10, which have been shown to induce NKG2A expression while inducing neutrophilia. ${ }^{42,43}$ Monalizumab is a humanized antibody targeting NKG2A and blocking its interaction with HLA-E thus preventing the propagation of inhibitory signals. It is currently in use for management of rheumatoid arthritis and several neoplastic disorders. ${ }^{44}$ It could represent a way for treatment of patients with severe COVID-19 infection, characterized by a sudden and marked reduction of the antiviral activity of NK and CD8+ cells . ${ }^{45,46}$
Our study has some limitations. First, this study was conducted on a small number of patients, thus the results should be interpreted with caution, and statistical non-significance may not rule out difference between severe and moderate cases. Second, the viremia profile of SARS-CoV-2 were not available, so further studies are needed to investigate the correlation between virus load kinetics and dynamics of cellular immune responses. Third we studied NK and CD8+T cells in the periphery, it would be more significant to study these subsets at primary sites of infection, lungs, and other tissue.

In conclusion, our results indicate that elevated NKG2A expression on cytotoxic lymphocytes correlates with disease severity in COVID-19 patients, and may serve as a potential marker for prognosis. Additionally, the blockade of NKG2A should be investigated as means of enhancing NK cell and cytotoxic T cells antiviral immunity in patients with severe COVID-19 infection.

\section{Author Contributions}

MY and NG assisted in the collection of samples and patients' data. MY contributed to laboratory work and analysis of data. ME and IS assisted in manuscript drafting and revision. All authors contributed significantly to the study's conception, design, and final approval of the manuscript.

\section{Declaration of Conflicting Interests}

The author(s) declared no potential conflicts of interest with respect to the research, authorship, and/or publication of this article.

\section{Funding}

This research did not receive any specific grant from funding agencies in the public, commercial, or notfor-profit sectors.

\section{Ethical approval}

The study was approved by the Ethical Committee of Ain Shams University Hospitals (03/09/2020).

\section{Informed consent}

A signed consent form was obtained from each study participant. 


\section{References}

1. Li, Q., Guan, X., Wu, P., et al. (2020a). Early transmission dynamics in Wuhan, China, of novel coronavirus-infected pneumonia. New England Journal of Medicine. 382, 1199-1207.

2. World Health Organization, (2021). WHO Coronavirus Disease (COVID-19) Dashboard. Available at https://covid19.who.int

3. Xu, Z., Li, S., Tian, S., et al. (2020a). Full spectrum of COVID-19 severity still being depicted. The Lancet. 395(10228), 947-948.

4. Huang, C., Wang, Y., Li, X., et al. (2020a). Clinical features of patients infected with 2019 novel coronavirus in Wuhan, China. Lancet. 395(10223), 497-506.

5. Zhong, J., Tang, J., Ye, C., and Dong, L. (2020). The immunology of COVID-19: is immune modulation an option for treatment? Lancet Rheumatol. 2(7),E428-E436.

6. André, P., Denis, C., Soulas, C., et al. (2018). AntiNKG2A $m A b$ is a checkpoint inhibitor that promotes anti-tumor immunity by unleashing both T and NK cells. Cell, 175(7), 1731-1743.

7. Haanen, J. B., \& Cerundolo, V. (2018). NKG2A, a New Kid on the Immune Checkpoint Block. Cell. 175(7), 1720-1722.

8. Zheng, M., Gao, Y., Wang, G et al. (2020a). Functional exhaustion of antiviral lymphocytes in COVID-19 patients. Cellular \& Molecular Immunology. 17(5), 533-535.

9. World Health Organization. (2020). Clinical management of COVID-19. Available at: https://www.who.int/publications/i/item/clinical -management-of-covid-19.

10. Tay, M. Z., Poh, C. M., Rénia, L. et al. (2020). The trinity of COVID-19: immunity, inflammation and intervention. Nature Reviews Immunology. 20(6), 363-374.

11.Zhang, W., Li, L., Liu, J., et al. (2020). The characteristics and predictive role of lymphocyte subsets in COVID-19 patients. International Journal of Infectious Diseases. 99, 92-99.

12. Wu, Y., Huang, X., Sun, J. et al. (2020). Clinical Characteristics and Immune Injury Mechanisms in 71 Patients with COVID-19. Msphere. 5(4),e00362-20.

13. Singh, K., Mittal, S., Gollapudi, S. et al. (2020). A meta-analysis of SARS-CoV-2 patients identifies the combinatorial significance of D-dimer, C-reactive protein, lymphocyte, and neutrophil values as a predictor of disease severity.
International Journal of Laboratory Hematology. Doi: 10.1111/ijlh.13354 [Epub ahead of print]

14. Guan, J., Wei, X., Qin, Set al. (2020). Continuous tracking of COVID-19 patients' immune status. International Immunopharmacology. 89, 107034.

15. Qin, C., Zhou, L., Hu, Z. et al. (2020). Dysregulation of immune response in patients with coronavirus 2019 (COVID-19) in Wuhan, China. Clinical Infectious Diseases. 71(15), 762768.

16. Li, M., Guo, W., Dong, Y. et al. (2020b). Elevated exhaustion levels of $\mathrm{NK}$ and $\mathrm{CD} 8+\mathrm{T}$ cells as indicators for progression and prognosis of COVID-19 disease. Frontiers in Immunology. 11.

17. Odak, I., Barros-Martins, J., Bošnjak, B., et al. (2020). Reappearance of effector $T$ cells is associated with recovery from COVID-19. EBioMedicine. 57, 102885.

18. Yang, L., Liu, S., Liu, J., et al. (2020). COVID-19: immunopathogenesis and Immunotherapeutics. Signal Transduction and Targeted Therapy. 5(1), 1-8.

19. Chen, G., Wu, D. I., Guo, W., et al. (2020a). Clinical and immunological features of severe and moderate coronavirus disease 2019. The Journal of Clinical Investigation. 130(5), 2620-2629.

20. Huang, I., Pranata, R., Lim, M. A., et al. (2020b). C-reactive protein, procalcitonin, D-dimer, and ferritin in severe coronavirus disease-2019: a meta-analysis. Therapeutic Advances in Respiratory Disease. 14, 1753466620937175.

21. Chaudhary, R., Garg, J., Houghton, D. E., et al. (2021). Thrombo-inflammatory Biomarkers in COVID-19: Systematic Review and Meta-analysis of 17,052 patients. Mayo Clinic Proceedings: Innovations, Quality \& Outcomes. Doi: https://doi.org/10.1016/j.mayocpiqo.2021.01.00 9.

22. Henry, B. M., De Oliveira, M. H. S., Benoit, S., et al. (2020). Hematologic, biochemical and immune biomarker abnormalities associated with severe illness and mortality in coronavirus disease 2019 (COVID-19): a meta-analysis. Clinical Chemistry and Laboratory Medicine . 58(7), 1021-1028.

23. Netea, M. G., Rovina, N., Koulouris, N., et al. (2020). Clinical and Translational Report Complex Immune Dysregulation in COVID-19 Patients with Severe Respiratory Failure II Clinical and Translational Report Complex Immune Dysregulation in COVID-19 Patients with Severe Respiratory Failure. Cell Host and Microbe. 27(6), 992-1000.e3. 
24. Wilk A. J., Rustagi A., Zhao N. Q., et al. (2020). A single-cell atlas of the peripheral immune response in patients with severe COVID-19. Nature Medicine. 26, 1070-1076.

25. Mazzoni, A., Salvati, L., Maggi, L., et al. (2020). Impaired immune cell cytotoxicity in severe COVID-19 is IL-6 dependent. The Journal of Clinical Investigation. 130(9) , 4694-4703.

26. Wang, F., Nie, J., Wang, H., et al. (2020). Characteristics of peripheral lymphocyte subset alteration in COVID-19 pneumonia. The Journal of Infectious Diseases. 221(11), 1762-1769.

27. Liao, M., Liu, Y., Yuan, J., et al. (2020). The landscape of lung bronchoalveolar immune cells in COVID-19 revealed by single-cell RNA sequencing. Nature Medicine. 26(6), 842-844.

28. Xiong, Y., Liu, Y., Cao, L., et al. (2020). Transcriptomic characteristics of bronchoalveolar lavage fluid and peripheral blood mononuclear cells in COVID-19 patients. Emerging Microbes \& Infections. 9, 761-770.

29. Demaria, O., Carvelli, J., Batista, L., et al. (2020). Identification of druggable inhibitory immune checkpoints on Natural Killer cells in COVID-19. Cellular \& Molecular Immunology. 17(9), 995997.

30. Carvelli, J., Demaria, O., Vély, F., et al. (2020). Identification of immune checkpoints in COVID19. Nat Res. Doi: 10.21203/rs.3.rs-27340.

31. Bortolotti, D., Gentili, V., Rizzo, S., et al. (2020). SARS-CoV-2 Spike 1 Protein Controls Natural Killer Cell Activation via the HLA-E/NKG2A Pathway. Cells.9(9), 1975.

32. Ahmed, F., Jo, D.-H., \& Lee, S.-H.,2020. Can Natural Killer Cells Be a Principal Player in AntiSARS-CoV-2 Immunity? Frontiers in Immunology. 11,586765 .

33. Market, M., Angka, L., Martel, A. B., et al. (2020). Flattening the COVID-19 curve with natural killer cell based immunotherapies. Frontiers in Immunology. 11,1512.

34. Nie, J., Li, Q., Wu, J., et al. (2020). Establishment and validation of a pseudovirus neutralization assay for SARS-CoV-2. Emerging Microbes \& Infections. 9(1), 680-686.

35. Zheng, H.-Y., Zhang, M., Yang, C.-X., et al. (2020b). Elevated exhaustion levels and reduced functional diversity of $\mathrm{T}$ cells in peripheral blood may predict severe progression in COVID-19 patients. Cellular \& Molecular Immunology. 17(5), 541-543.

36. Diao, B., Wang, C., Tan, Y., et al. (2020). Reduction and Functional Exhaustion of T Cells in Patients With Coronavirus Disease 2019 (COVID19). Frontiers in Immunology. 11.

37. Xu, Z., Shi, L., Wang,Y., et al. (2020b). Pathological findings of COVID-19 associated with acute respiratory distress syndrome. Lancet Respir Med. 8(4):420-422.

38. Antonioli, L., Fornai, M., Pellegrini, C., et al. (2020). NKG2A and COVID-19: another brick in the wall. Cellular \& Molecular Immunology. 17,672-4.

39. van Eeden, C., Khan, L., Osman, M. S., et al. (2020). Natural killer cell dysfunction and its role in COVID-19. International Journal of Molecular Sciences, 21(17), 6351.

40. Nandi, B., \& Behar, S. M., (2011). Regulation of neutrophils by interferon- $\nu$ limits lung inflammation during tuberculosis infection. Journal of Experimental Medicine. 208(11), 22512262.

41. Jewett, A., (2020). The potential effect of novel coronavirus SARS-CoV-2 on NK cells; a perspective on potential therapeutic interventions. Frontiers in Immunology. 11, 1692.

42. Manickam, C., Sugawara, S., \& Reeves, R. K., (2020). Friends or foes? The knowns and unknowns of natural killer cell biology in COVID19 and other coronaviruses. PLoS Pathogens. 16(8), e1008820.

43. Masselli, E., Vaccarezza, M., Carubbi, C., et al. (2020). NK cells: A double edge sword against SARS-CoV-2. Advances in Biological Regulation. 77, 100737.

44. van Hall, T., André, P., Horowitz, A., et al. (2019). Monalizumab: inhibiting the novel immune checkpoint NKG2A. Journal for ImmunoTherapy of Cancer. 7, 263.

45. Yaqinuddin, A., \& Kashir, J., (2020). Innate immunity in COVID-19 patients mediated by NKG2A receptors, and potential treatment using Monalizumab, Cholroquine, and antiviral agents. Medical Hypotheses. 140, 109777.

46. Odabasi, Z., \& Cinel, I., (2020). Consideration of severe coronavirus disease 2019 as viral sepsis and potential use of immune checkpoint inhibitors. Critical Care Explorations. 2(6), e0141. 